\title{
Direct Inductive Sensor-to-Microcontroller Interface
}

\section{Circuit}

Authors: Zivko Kokolanski ${ }^{\mathrm{a}}$, Josep Jordana ${ }^{\mathrm{b}}$, Manel Gasulla ${ }^{\mathrm{b}}$, Vladimir Dimcev ${ }^{\mathrm{a}}$ and Ferran Reverter $^{\mathrm{b}}$

Address: $\quad{ }^{a}$ Ss. Cyril and Methodius University

Ruger Boskovic bb, 1000 Skopje, Republic of Macedonia

b e-CAT Research Group

Department of Electronic Engineering

Universitat Politècnica de Catalunya (UPC) - BarcelonaTech

C/ Esteve Terradas 7, C4, 08860 Castelldefels (Barcelona), Spain

Corresponding author: $\quad$ Ferran Reverter, ferran.reverter@upc.edu

Phone: +34 934137076

Telefax: +34 934137007 
Abstract-This paper proposes and analyses a microcontroller-based interface circuit for inductive sensors with a variable self-inductance. Besides the microcontroller $(\mu \mathrm{C})$ and the sensor, the circuit just requires an external resistor and a reference inductor so that two RL circuits are formed. The $\mu \mathrm{C}$ appropriately excites such RL circuits in order to measure the discharging time of the voltage across each inductor (i.e. sensing and reference) and then it uses such discharging times to estimate the sensor inductance. Experimental tests using different commercial $\mu \mathrm{Cs}$ at different clock frequencies show the limitations (especially, due to parasitic resistances and quantisation) and the performance of the proposed circuit when measuring inductances in the millihenry range. A non-linearity error lower than $0.3 \%$ FullScale Span (FSS) and a resolution of 10 bits are achieved, which are remarkable values considering the simplicity of the circuit.

Keywords: Inductive sensor; microcontroller; sensor electronic interface. 


\section{INTRODUCTION}

Inductive sensors are widely used in industry electronic instrumentation because they are robust and compact and, in comparison to capacitive sensors, they are less affected by environmental factors such as humidity and dust [1]. Within the group of inductive sensors, those based on a variable self-inductance (due to changes of either the magnetic reluctance or the number of turns of the coil winding) are quite common to measure displacement (e.g. of $\pm 1 \mathrm{~mm}[2], \pm 5 \mathrm{~mm} \mathrm{[3]} \mathrm{and} \pm 6 \mathrm{~mm}$ [4]), position (e.g. of a piston inside a power cylinder [5]) and proximity. Other physical quantities that indirectly cause a displacement are also measured through these sensors, for instance: inductive pressure sensors based on either a Bourdon tube [6] or a vertical coil embedded into an integrated circuit (IC) package [7]. Inductive sensors have also been proposed to measure temperature by using cores with a low Curie temperature [8]. In the previous applications $[3,4,6,7]$, the sensor inductance is in the range of units or tens of millihenry, but lower values (e.g. units or tens of microhenry) can also be found.

Two main types of interface circuits have been proposed for inductive sensors with a variable self-inductance and both usually operate at low-medium frequencies. The first type is a relaxation oscillator (based, for instance, on a 555 IC timer [7]) providing a time-modulated signal that can be read by a digital system (e.g. a $\mu \mathrm{C}$ with an embedded timer) without using an analog-to-digital converter (ADC). The second type is an AC-excited bridge (such as the Maxwell bridge [6] or the Maxwell-Wien bridge [3]) providing an amplitude-modulated signal that needs to be demodulated and digitized before being read by the digital system. The reference inductor usually required in bridge circuits is proposed to be emulated by a generalized impedance converter in [9]. Another interface circuit suggested for inductive sensors that does not belong to the previous two groups is the dual slope inductance-to-digital converter whose output can be read by a digital system without an ADC, as in the oscillator 
circuits, but it needs operational amplifiers (OpAmp) and analogue comparators in the signal path [4]. There are also a few commercial ICs (e.g. LDC1000 from Texas Instruments) that perform an inductance-to-digital conversion, but these are mainly intended for inductive sensors based on eddy current operating at higher frequencies.

With the aim of reducing the cost and power consumption of sensor electronic interfaces, the concept of "direct interface circuit" has been widely proposed, analysed and tested for resistive [10-12] and capacitive [13-15] sensors. In these circuits, the sensor resistance (or capacitance) together with a capacitor (or resistor) form an $\mathrm{RC}$ circuit whose charging or discharging time is directly measured by a $\mu \mathrm{C}$ through an embedded digital timer and without using any intermediate active circuit (such as comparators, OpAmps, timers and/or ADC). The performance of such circuits is quite remarkable taking into account their simplicity, for instance: a non-linearity error (NLE) of $0.01 \%$ full-scale span (FSS) and an effective resolution of 13 bits when measuring resistive sensors in the kiloohm range [10,11], and $0.1 \%$ FSS and 9 bits when measuring capacitive sensors in the picofarad range [13]. Although the same operating principle could be applied to measure inductive sensors by employing an RL circuit, instead of an RC circuit, formed by the sensor inductance and a resistor, no attempts to do so have been reported so far. Just in $[16,17]$ we can find very preliminary circuit proposals but these have not been either analysed or tested.

As a continuation of the work presented in $[18,19]$, this paper proposes, theoretically analyses and experimentally evaluates a direct interface circuit for inductive sensors with a variable self-inductance. In the proposed circuit, the inductive sensor is excited by a single step pulse and the result of the measurement is the inductance value at low frequencies; this is assuming that the frequency dependence of the inductance (due to the frequency dependence of the permeability) starts decreasing at high enough frequencies. For this reason, the proposed circuit is not suitable for those inductive sensors whose operating principle involves the measurement of the inductance at medium-high frequencies; this is the case, for instance, 
of eddy-current sensors that are generally excited by an AC signal of high frequency (say, units or tens of $\mathrm{MHz}$ ) so as to have an appropriate penetration depth in the metallic target to be detected [20, 21]. Furthermore, the sensor is expected to have an inductance of some units or tens of millihenry $[3,4,6,7]$; lower values of inductance would require a very high speed reference oscillator that is not feasible nowadays in common low-cost 8-bit microcontrollers.

The paper is organised as follows: Section 2 describes the operating principle of the circuit, Section 3 analyses the error sources, Section 4 shows experimental results, and Section 5 provides the main conclusions.

\section{OPERATING PRINCIPLE}

The proposed direct interface circuit for inductive sensors is shown in Fig. 1a. Besides the $\mu \mathrm{C}$ and the sensor $\left(L_{x}\right)$, this electronic interface just needs a reference inductor $\left(L_{\mathrm{r}}\right)$ that is used for a single-point calibration, and an external resistor $\left(R_{0}\right)$ that limits the current provided by the $\mu \mathrm{C}$. With these components, two RL circuits are formed: $R_{0}$ together with $L_{x}$, and $R_{0}$ together with $L_{\mathrm{r}}$. Then, each RL circuit is appropriately excited by the $\mu \mathrm{C}$ so as to measure the discharging time ( $T_{x}$ and $T_{\mathrm{r}}$ ) of the voltage across each inductor ( $L_{x}$ and $L_{\mathrm{r}}$, respectively). As for the $\mu \mathrm{C}$, only digital peripherals (specifically, a timer) and digital input/output ports are required, thus resulting in a fully-digital sensor interface circuit.

The measurement of the discharging time of each RL circuit requires two phases. Figs. 1c and $1 \mathrm{~d}$ show, respectively, the state of the digital ports of the $\mu \mathrm{C}$ during the first and second phase when measuring the RL circuit that includes $L_{x}$. In the first phase (Fig. 1c), pin 1 generates a step pulse (i.e. from a digital ' 0 ' to ' 1 ', or from $0 \mathrm{~V}$ to the supply voltage, $V_{\mathrm{DD}}$ ) that is synchronized with the start of the timer, pin 3 provides a digital ' 0 ' (i.e. $0 \mathrm{~V}$ ), and pins 2 and 4 are in a high-impedance (HZ) state. This configuration results in a discharging voltage across $L_{x}$, as shown in Fig. 1b, that is monitored by pin 2. When such a discharging voltage reaches the low threshold voltage $\left(V_{\mathrm{TL}}\right)$ of the digital Schmitt-trigger $(\mathrm{ST})$ buffer embedded 
into pin 2, the timer stops and a digital number with information about the length of $T_{x}$ is registered. Under ideal conditions, $T_{x}$ is equal to

$$
T_{x}=\frac{L_{x}}{R_{0}} \ln \left(\frac{V_{\mathrm{DD}}}{V_{\mathrm{TL}}}\right)
$$

In the second phase (Fig. 1d), pin 1 provides a digital ' 0 ', whereas the other pins do not change their state. With this configuration, the inductor current is discharged towards zero; this phase must be long enough (at least five times the discharging time constant) so as to be sure that the energy stored before in the inductor is removed. Afterwards, the circuit operates similarly for the measurement of the RL circuit that includes $L_{\mathrm{r}}$, but pin 3 is in HZ state and pin 4 provides a digital ' 0 '. In that case, the result is a digital number with information about the length of $T_{\mathrm{r}}$, which is ideally equal to

$$
T_{\mathrm{r}}=\frac{L_{\mathrm{r}}}{R_{0}} \ln \left(\frac{V_{\mathrm{DD}}}{V_{\mathrm{TL}}}\right)
$$

After measuring $T_{x}$ and $T_{\mathrm{r}}$, the following single-point calibration technique is proposed to be applied

$$
L_{x}^{*}=\frac{T_{x}}{T_{\mathrm{r}}} L_{\mathrm{r}}
$$

where $L_{x}^{*}$ is the estimated value of $L_{x}$. Replacing (1) and (2) in (3) yields $L_{x}^{*}=L_{x}$ and, hence, the estimated value has no error under ideal conditions. Furthermore, changes of temperature affecting the circuit are cancelled out by (3) whenever (i) temperature remains constant during the measurement of $T_{x}$ and $T_{\mathrm{r}}$, and (ii) the reference inductor has a low temperature coefficient. Note that changes of temperature can affect the values of $R_{0}, V_{\mathrm{DD}}$ and $V_{\mathrm{TL}}$ in (1) and (2), but if they do in the same way in both measurements, then such thermal effects are compensated through (3). Time drifts affecting $R_{0}, V_{\mathrm{DD}}$ and $V_{\mathrm{TL}}$ are also auto-calibrated by (3). In order to compensate for the temperature dependence and time drifts of $L_{x}$, the circuit would require an $L_{\mathrm{r}}$ with the same dependence. The application of a three-signal calibration technique $[10,13]$ seems in principle unnecessary since the offset parasitic inductance (of 
some units of nanohenry) introduced by the circuit itself (for instance, due to the interconnections on the printed circuit board or to the bonding pad of the $\mu \mathrm{C}$ chip) is much lower than the sensor inductance (of some units or tens of millihenry).

The current consumption of the proposed circuit can be reduced by following the guidelines suggested in [22]. In the first phase, the discharging time should be measured by the timer running at high frequency (e.g. units or tens of $\mathrm{MHz}$ ) so as to have a good timing resolution, but the CPU (Central Processing Unit) should be off whenever this does not stop the interrupt system and the timer. In the second phase, just the CPU should be on but running at low frequency (e.g. tens or hundreds of $\mathrm{kHz}$ ) since we do not need an accurate control of the duration of that phase. Using such configurations, the current consumption of the internal electronics of the $\mu \mathrm{C}$ is about ten times higher in the first phase [22]. On the other hand, we also have the current flowing through the external RL circuit, but only that required in the first phase must be considered in terms of current consumption since the RL circuit is disconnected from $V_{\mathrm{DD}}$ in the second phase. Accordingly, the average current consumption in active mode (i.e. when the $\mu \mathrm{C}$ is working to carry out the measurements) can be approximated to $I_{\mathrm{T}} \approx\left(I_{\mathrm{int}}+I_{\mathrm{ext}}\right)\left(T_{\mathrm{p} 1} /\left(T_{\mathrm{p} 1}+T_{\mathrm{p} 2}\right)\right)$, where $I_{\text {int }}$ and $I_{\mathrm{ext}}$ are the average current consumed by, respectively, the internal electronics and the external $\mathrm{RL}$ circuit in the first phase, and $T_{\mathrm{p} 1}$ and $T_{\mathrm{p} 2}$ are the lengths of the first and second phases, respectively. Assuming $I_{\mathrm{int}}=4 \mathrm{~mA}$ at $20 \mathrm{MHz}$ [22], $I_{\mathrm{ext}}=0.37 \cdot V_{\mathrm{DD}} / R_{0}$, and $T_{\mathrm{p} 2}=5 \cdot T_{\mathrm{p} 1}$ (so as to remove the energy stored in the inductor), then $I_{\mathrm{T}} \approx 2 \mathrm{~mA}$ at $V_{\mathrm{DD}}=5 \mathrm{~V}$ and, therefore, the power consumption is $10 \mathrm{~mW}$; it has been assumed that (i) the discharging time is almost equal to the time constant and, hence, the factor 0.37 in the previous equation, and (ii) the equivalent resistance is $200 \Omega$, which considers the values of the external resistor and the parasitic resistances reported later in Section 4. 


\section{ERROR ANALYSIS}

As indicated before, under ideal conditions, we have $L_{x}^{*}=L_{x}$ when (3) is applied. However, in practice, the estimated value undergoes some errors mainly due to (i) the parasitic resistances of both the inductors and the digital ports of the $\mu \mathrm{C}$, and (ii) the quantisation of the discharging-time measurement.

\section{A. Effect of parasitic resistances}

The effect of parasitic resistances on the proposed direct interface circuit are analysed using the equivalent circuit shown in Fig. 2, where $R_{\mathrm{p}, 1}$ and $R_{\mathrm{n}, 1}$ are the parasitic output resistances of pin 1 when it provides a digital ' 1 ' and ' 0 ', respectively, $R_{\mathrm{n}, 3}$ and $R_{\mathrm{n}, 4}$ are the parasitic output resistances of pins 3 and 4 , respectively, when they provide a digital ' 0 ', $Z_{\text {in }}$ is the input impedance (which is assumed to be very high) of a pin set in $\mathrm{HZ}$ state, and $R_{x}$ and $R_{\mathrm{r}}$ are the parasitic equivalent series resistance (ESR) of $L_{x}$ and $L_{\mathrm{r}}$, respectively. The different states of the digital ports are modelled by switches $\left(\mathrm{S}_{1}, \mathrm{~S}_{3}\right.$ and $\left.\mathrm{S}_{4}\right)$.

Assuming the parasitic resistances shown in Fig. 2, the discharging times $T_{x}$ and $T_{\mathrm{r}}$ are, respectively, equal to

$$
\begin{aligned}
& T_{x}=\frac{L_{x}}{R_{\mathrm{p}, 1}+R_{0}+R_{3}} \ln \left[\frac{\left(R_{\mathrm{p}, 1}+R_{0}\right) V_{\mathrm{DD}}}{\left(R_{\mathrm{p}, 1}+R_{0}+R_{3}\right) V_{\mathrm{TL}}-R_{3} V_{\mathrm{DD}}}\right] \\
& T_{\mathrm{r}}=\frac{L_{\mathrm{r}}}{R_{\mathrm{p}, 1}+R_{0}+R_{4}} \ln \left[\frac{\left(R_{\mathrm{p}, 1}+R_{0}\right) V_{\mathrm{DD}}}{\left(R_{\mathrm{p}, 1}+R_{0}+R_{4}\right) V_{\mathrm{TL}}-R_{4} V_{\mathrm{DD}}}\right]
\end{aligned}
$$

where $R_{3}=R_{\mathrm{n}, 3}+R_{x}$ and $R_{4}=R_{\mathrm{n}, 4}+R_{\mathrm{r}}$. Replacing now (4) and (5) in (3) yields the actual relation between $L_{x}^{*}$ and $L_{x}$ : 


$$
L_{x}^{*}=\frac{1+\frac{R_{4}}{R_{\mathrm{p}, 1}+R_{0}}}{1+\frac{R_{3}}{R_{\mathrm{p}, 1}+R_{0}}} \frac{\ln \left[\frac{V_{\mathrm{DD}}}{V_{\mathrm{TL}}+\left(V_{\mathrm{TL}}-V_{\mathrm{DD}}\right) \frac{R_{3}}{R_{\mathrm{p}, 1}+R_{0}}}\right]}{\left[\frac{V_{\mathrm{DD}}}{V_{\mathrm{TL}}+\left(V_{\mathrm{TL}}-V_{\mathrm{DD}}\right) \frac{R_{4}}{R_{\mathrm{p}, 1}+R_{0}}}\right]} L_{x}
$$

From (6), if $R_{3}=R_{4}$ then $L_{x}^{*}=L_{x}$. However, if the parasitic resistances are not well-matched (basically due to the mismatch between $R_{x}$ and $R_{\mathrm{r}}$ since the mismatch between $R_{\mathrm{n}, 3}$ and $R_{\mathrm{n}, 4}$ is expected to be just a few tenths of ohm [10]), then we can achieve $L_{x}^{*} \approx L_{x}$ by having $\left(R_{\mathrm{p}, 1}+R_{0}\right) \gg>R_{3}$ and $\left(R_{\mathrm{p}, 1}+R_{0}\right)>>R_{4}$. Therefore, the higher the value of $R_{0}$, the lower the error due to parasitic resistances. Nevertheless, as shown later in the next subsection, a highvalue $R_{0}$ is not advisable in terms of quantisation effects.

Considering $\left(R_{\mathrm{p}, 1}+R_{0}\right) \gg>R_{3}$ and $\left(R_{\mathrm{p}, 1}+R_{0}\right)>>R_{4}$ and applying first-order Taylor-series approximations, Eq. (6) can be simplified to

$$
L_{x}^{*} \approx\left[1+\alpha \Delta R_{34}\right] L_{x}
$$

where

$$
\alpha=\frac{1}{R_{\mathrm{p}, 1}+R_{0}}\left[\frac{\frac{V_{\mathrm{DD}}}{V_{\mathrm{TL}}}-1}{\ln \left(\frac{V_{\mathrm{DD}}}{V_{\mathrm{TL}}}\right)}-1\right]
$$

and $\Delta R_{34}=R_{3}-R_{4}$. According to (7), the mismatch between parasitic resistances brings about a gain error that is positive (i.e. slope higher than 1) if $\Delta R_{34}>0$, but it is negative (i.e. slope lower than 1) if $\Delta R_{34}<0$. On the other hand, the relative error due to parasitic resistances can be expressed, from (7), as

$$
e_{\mathrm{r}}=\frac{L_{x}^{*}-L_{x}}{L_{x}}=\alpha \Delta R_{34}
$$


Assuming the experimental data presented later in Table I for the different variables involved in (8), we have $\alpha \approx 0.005 \Omega^{-1}$ that would cause a relative error of $0.5 \%$ for $\Delta R_{34}=1 \Omega$.

Some inductive sensors (e.g. those based on the variation of the number of turns of the coil winding) may offer an $R_{x}$ that changes with the value of $L_{x}$. In that case, $R_{x}$ can be expressed in a first approximation as

$$
R_{x}=R_{x, 0}+\beta L_{x}
$$

where $R_{x, 0}$ is the parasitic ESR when $L_{x}=0$, and $\beta$ is a sensitivity factor in $\Omega / \mathrm{mH}$. Consequently, the resistance mismatch also depends on $L_{x}$ as

$$
\Delta R_{34}=\Delta R_{34,0}+\beta L_{x}
$$

where $\Delta R_{34,0}=R_{\mathrm{n}, 3}+R_{x, 0}-R_{\mathrm{n}, 4}-R_{\mathrm{r}}$. Replacing now (11) in (7) yields

$$
L_{x}^{*} \approx\left[1+\alpha \Delta R_{34,0}\right] L_{x}+\alpha \beta L_{x}^{2}
$$

which shows a quadratic relation between $L_{x}^{*}$ and $L_{x}$, thus causing a non-linearity error. Note that the electronic circuit is auto-calibrated by (3), but the overall measurement system (i.e. the sensor with the electronics) is expected to be subjected later to a two-point field calibration (for instance, two well-known displacements will be applied and the output values will be registered) and, therefore, the non-linearity error becomes the main source of inaccuracy.

\section{B. Effect of quantisation}

The time-to-digital conversion performed by the digital timer brings about a quantisation error in the measurement of $T_{x}$ and $T_{\mathrm{r}}$. If an input associated with a capture module is used to monitor the discharging voltage (i.e. pin 2 in Fig. 1a), the quantisation error ranges from $-T_{\mathrm{s}}$ $\left(=1 / f_{\mathrm{s}}\right)$ to $0[11]$, where $T_{\mathrm{s}}$ and $f_{\mathrm{s}}$ are, respectively, the period and the frequency of the reference oscillator of the timer. If then the quantified values of $T_{x}$ and $T_{\mathrm{r}}$ (i.e. $T_{x, \mathrm{q}}$ and $T_{\mathrm{r}, \mathrm{q}}$, 
respectively) are used in (3), the estimation of the sensor inductance undergoes an error. The worst cases, assuming $\Delta R_{34}=0$, are when:

(a) $T_{x}$ suffers from the minimum quantisation error (i.e. zero), whereas $T_{\mathrm{r}}$ suffers from the maximum one (i.e. $-T_{\mathrm{s}}$ ), thus resulting in

$$
L_{x}^{*}=\frac{T_{x, \mathrm{q}}}{T_{\mathrm{r}, \mathrm{q}}} L_{\mathrm{r}}=\frac{T_{x}}{T_{\mathrm{r}}-T_{\mathrm{s}}} L_{\mathrm{r}} \approx L_{\mathrm{x}}\left(1+\frac{T_{\mathrm{s}}}{T_{\mathrm{r}}}\right)
$$

which behaves as a gain error.

(b) $T_{x}$ suffers from the maximum quantisation error (i.e. $-T_{\mathrm{s}}$ ), whereas $T_{\mathrm{r}}$ suffers from the minimum one (i.e. zero), thus resulting in

$$
L_{x}^{*}=\frac{T_{x, \mathrm{q}}}{T_{\mathrm{r}, \mathrm{q}}} L_{\mathrm{r}}=\frac{T_{x}-T_{\mathrm{s}}}{T_{\mathrm{r}}} L_{\mathrm{r}}=L_{\mathrm{x}}-\frac{T_{\mathrm{s}}}{T_{\mathrm{r}}} L_{\mathrm{r}}
$$

which behaves as an offset error.

Equations (13) and (14) define, respectively, the high and low boundaries of the response $L_{x}^{*}$ versus $L_{x}$ due to quantisation effects, as shown in Fig. 3. Considering that the values of $L_{x}^{*}$ are randomly distributed between those boundaries (see, for example, the crosses in Fig. 3), then the most critical error introduced by quantisation seems to be the non-linearity. The closer the boundaries, the lower the non-linearity error, and this can be achieved by (i) a fast reference oscillator (i.e. a lower value of $T_{\mathrm{s}}$ ), although this increases the current consumption of the $\mu \mathrm{C}$, and (ii) a low-value $R_{0}$, which increases, from (2), the value of $T_{\mathrm{r}}$. Note, however, that the value of $R_{0}$ can be as low as required whenever it limits, together with the parasitic resistances, the current of the RL circuit below the maximum current that can be sourced/sunk by the digital ports of the $\mu \mathrm{C}$. On the other hand, the relative error due to quantisation is determined by

$$
-\frac{T_{\mathrm{s}}}{T_{\mathrm{r}}} \frac{L_{\mathrm{r}}}{L_{x}} \leq e_{\mathrm{r}} \leq \frac{T_{\mathrm{s}}}{T_{\mathrm{r}}}
$$

which, as in the non-linearity error, can be reduced by decreasing the factor $T_{\mathrm{s}} / T_{\mathrm{r}}$. 


\section{Other effects}

The parasitic capacitances $\left(C_{\mathrm{p}}\right)$ of the digital input ports of the $\mu \mathrm{C}$, such as pin 2 in Fig. 1a, could limit the speed of the transient response of the voltage at pin 2 at the beginning of the first phase (see Fig. 1b). However, assuming a common value of $C_{\mathrm{p}}$ (say, $10 \mathrm{pF}$ [14]) and $R_{0}=100 \Omega$ (see Section 4), the settling time of such a transient response is $5 \mathrm{~ns}$, which is much lower than the minimum period (i.e. $1 / 16 \mathrm{MHz}=62 \mathrm{~ns}$ ) used later for the reference oscillator of the timer and, therefore, the effect of $C_{\mathrm{p}}$ on the discharging-time measurement can be assumed negligible. Similar effects are expected to be caused by the parasitic capacitance of the inductors, i.e. a capacitance in parallel with $L_{\mathrm{x}}$ or $L_{\mathrm{r}}$. The effect of leakage currents of the digital ports is also expected to be insignificant, at least in CMOS microcontrollers.

Another source of error could be the frequency dependence of the relative permeability $\left(\mu_{\mathrm{r}}\right)$ of the ferromagnetic material used in the core of the inductors. A Cole-Cole plot of $\mu_{\mathrm{r}}$ tends to be a semicircle [23] that remembers the typical response of a low-pass system with a dominant pole. Therefore, in a first approximation and in the Laplace domain, the frequency dependence of $\mu_{\mathrm{r}}$ can be expressed as $\mu_{\mathrm{r}}(s)=\mu_{\mathrm{r}, 0}\left(1+\omega_{\mathrm{c}}^{-1} s\right)^{-1}$, where $\mu_{\mathrm{r}, 0}$ is the permeability at $s=0$ and $\omega_{\mathrm{c}}\left(=2 \pi f_{\mathrm{c}}\right)$ is the frequency of the dominant pole where the real and imaginary parts of the complex permeability are equal. Accordingly, the inductance also depends on frequency as $L(s)=L_{0}\left(1+\omega_{\mathrm{c}}^{-1} s\right)^{-1}$, where $L_{0}$ is the inductance at $s=0$. If now the RL circuit in Fig. 1a is analysed considering such frequency dependence of $L$, then the resulting discharging time to be measured is

$$
T=\left(L_{0} / R_{0}+\omega_{\mathrm{c}}^{-1}\right) \ln \left(\frac{V_{\mathrm{DD}}}{V_{\mathrm{TL}}} \frac{L_{0} / R_{0}}{L_{0} / R_{0}+\omega_{\mathrm{c}}^{-1}}\right)
$$

Comparing (16) with (1) or (2), we can see that there is an error in the discharging time that depends on $\omega_{\mathrm{c}}$; the higher the value of $\omega_{\mathrm{c}}$, the lower the error. In order to have an error lower 
than the minimum period used later for the reference oscillator of the timer (i.e. $62 \mathrm{~ns}$ ), $f_{\mathrm{c}}$ should be higher than $500 \mathrm{kHz}$ at $V_{\mathrm{DD}}=5 \mathrm{~V}, V_{\mathrm{TL}}=1.50 \mathrm{~V}$ and $R_{0}=100 \Omega$, and higher than $700 \mathrm{kHz}$ at $V_{\mathrm{DD}}=5 \mathrm{~V}, V_{\mathrm{TL}}=2.42 \mathrm{~V}$ and $R_{0}=100 \Omega$, which are the two test conditions considered in Section 4. Note that a ferrite core or a powdered-iron core generally has an $f_{\mathrm{c}}$ higher than $1 \mathrm{MHz}$ [24] and, therefore, the effects on the discharging-time measurement are expected to be negligible, at least for these ferromagnetic materials.

\section{EXPERIMENTAL RESULTS AND DISCUSSION}

The direct interface circuit shown in Fig. 1a has been implemented using two commercial 8-bit CMOS microcontrollers (PIC16F877 and AVR ATmega328P) whose main features are summarised in Table I; the output resistance of the digital ports were measured using the method proposed in [10]. The PIC ran on a 20-MHz oscillator, but $f_{\mathrm{s}}$ was four times lower (i.e. $5 \mathrm{MHz}$ ); an internal prescaler rate of 2 was also used to assess the effects of operating at a lower frequency (to be precise, $2.5 \mathrm{MHz}$ ). On the other hand, the AVR ran on a 16-MHz oscillator providing $f_{\mathrm{s}}=16 \mathrm{MHz}$. The discharging voltage was monitored by a digital input (i.e. pin 2 in Fig. 1a) associated with a capture module that automatically registers the value of the timer when a falling edge is detected. The inductance values corresponding to $L_{x}$ (between $1 \mathrm{mH}$ and $100 \mathrm{mH}$ ) were provided by a variable decade inductance box (Metrel MA 2705) and the actual value of those inductances was measured by an RCL meter (Philips PM6303A). The parasitic resistance $\left(R_{x}\right)$ depended on $L_{x}$ as $R_{x}[\Omega]=0.343+0.062 L_{x}[\mathrm{mH}]$ in the range from $1 \mathrm{mH}$ to $10 \mathrm{mH}$, and as $R_{x}[\Omega]=1.233+0.043 L_{x}[\mathrm{mH}]$ in the range from $10 \mathrm{mH}$ to $100 \mathrm{mH}$. The reference inductor had a nominal value of $1.2 \mathrm{mH}$, but the actual values were $L_{\mathrm{r}}=1.192 \mathrm{mH}$ and $R_{\mathrm{r}}=2.415 \Omega$; note that higher values of $L_{\mathrm{r}}$ (e.g. $10 \mathrm{mH}$ or $50 \mathrm{mH}$ ) would result in a bulky and expensive component. In order to decrease the effects of quantisation, we employed a low-value $R_{0}$ with a nominal (actual) value of $100 \Omega\left(99.0 \Omega\right.$ ). This value of $R_{0}$ together with the parasitic resistances were high enough to limit the current sourced/sunk by 
the digital ports, whose maximum value is $25 \mathrm{~mA}$ for the PIC and $40 \mathrm{~mA}$ for the AVR. All measurements were carried out at room temperature.

Using the previous components (to be precise, the PIC microcontroller), the transient response of the voltage at pin 2 during the discharging-time measurement was acquired by a digital oscilloscope, as shown in Fig. 4. In comparison with the theoretical transient response represented in Fig. 1b, there was a significant difference at the beginning of the second phase since the ESD (Electrostatic Discharge) protection diode embedded into pin 2 became forward biased and, then, the voltage was limited to around $-0.7 \mathrm{~V}$. However, this is not critical for the operating principle of the circuit because the discharging time is measured before the ESD diode becomes forward biased.

The experimental results when measuring inductances from $1 \mathrm{mH}$ to $10 \mathrm{mH}$ are shown in Fig. 5 for three different cases: (a) PIC with $f_{\mathrm{s}}=2.5 \mathrm{MHz}$, (b) PIC with $f_{\mathrm{s}}=5 \mathrm{MHz}$, and (c) AVR with $f_{\mathrm{s}}=16 \mathrm{MHz}$. Each figure shows the experimental values of $L_{x}^{*}$ versus $L_{x}$, and the NLE calculated by fitting a straight line to the experimental data using the least-squares method and then expressed as a percentage of the FSS; the value of $L_{x}^{*}$ represented in Fig. 5 is the mean of thirty measurements. The results in Fig. 5 suggest that the effects of quantisation predominate over those of parasitic resistances because: (i) the NLE is quite random in terms of sign and magnitude, and (ii) the maximum value of the NLE (i.e. $0.70 \%,-0.23 \%$ and $0.12 \%$ in cases (a), (b) and (c), respectively) decreases with the speed of the reference oscillator of the timer, which is probably due to the fact that the boundaries in Fig. 3 were closer. Since the discharging-time measurement was mainly affected by quantisation, the digital number with information about $T_{x}$ was very stable for a given value of $L_{x}$ and, hence, averaging did not bring any benefit. In such conditions, a resolution of 8, 9, and 10 bits was possible using the PIC at $2.5 \mathrm{MHz}$, PIC at $5 \mathrm{MHz}$, and AVR at $16 \mathrm{MHz}$, respectively, for an overall measuring time of a few hundreds of microsecond. 
Figure 6 shows the experimental results when measuring inductances from $10 \mathrm{mH}$ to $100 \mathrm{mH}$ for the same three cases indicated before. Again, each figure shows the experimental values of $L_{x}^{*}$ versus $L_{x}$, and the resulting NLE. Unlike what happens in Fig. 5, the results in Fig. 6 suggest that the effects of parasitic resistances predominate over those of quantisation since: (i) the NLE shows the typical response generated by a quadratic relation, as predicted by (12), and (ii) the maximum value of the NLE (i.e. $0.26 \%, 0.28 \%$ and $0.22 \%$ in cases (a), (b) and (c), respectively) is quite independent of the speed of the reference oscillator of the timer. Table II shows, for the three cases under test, the theoretical value of the coefficients of the quadratic response obtained from (12) using the data in Table I, and the experimental value of those coefficients that result from fitting a quadratic polynomial to the experimental data in Fig. 6. The agreement between those coefficients reinforces the idea that the parasitic resistances seem to be the dominating error source. Moreover, in such a range of inductances, the discharging-time measurement was affected by trigger noise [11] so that the digital number with information about $T_{x}$ had some variability for a given value of $L_{x}$. The standard deviation of $L_{x}^{*}$ was about $70 \mu \mathrm{H}$ for the PIC, which corresponds to an effective resolution of 10.5 bits for an overall measuring time of a few units of millisecond. For the AVR, the standard deviation was higher probably due to a higher level of noise generated by the higher frequency of the reference oscillator of the timer.

\section{CONCLUSIONS}

This work has gone a step further in the field of direct interface circuits by proposing, analysing and testing a circuit for inductive sensors with a variable self-inductance. The proposed circuit uses a low-cost $\mu \mathrm{C}$ to measure the discharging time of two RL circuits formed by the sensor inductance, a reference inductor and an external resistor. The sensor inductance is then estimated through a single-point calibration that applies such discharging times. Two main error sources have been identified: the quantisation of the discharging-time measurement and the parasitic resistances of both the inductors and the digital ports of the $\mu \mathrm{C}$. 
Experimental results with different commercial $\mu \mathrm{Cs}$ at different speeds have shown that the effects of quantisation predominate when measuring inductances from $1 \mathrm{mH}$ to $10 \mathrm{mH}$, whereas those of parasitic resistances are more significant from $10 \mathrm{mH}$ to $100 \mathrm{mH}$. In both ranges, however, it is feasible to achieve a NLE lower than $0.3 \%$ FSS and a resolution of 10 bits, which are remarkable values considering the simplicity of the proposed circuit. 


\section{REFERENCES}

[1] K. Antonelli et al., Displacement measurement, linear and angular (Chapter 6), in: J.G. Webster, The measurement, instrumentation and sensors handbook, CRC Press, Boca Raton, Florida, 1999.

[2] A. Flammini, D. Marioli, E. Sisinni, A. Taroni, A multichannel DSP-based instrument for displacement measurement using differential variable reluctance transducer, IEEE Trans. Instrum. Meas. 54 (1) (2005) 178-183.

[3] S. Chattopadhyay, S.C. Bera, Modification of the Maxwell-Wien bridge for accurate measurement of a process variable by an inductive transducer, IEEE Trans. Instrum. Meas. 59 (9) (2010) 2445-2449.

[4] N. Philip, B. George, Design and Analysis of a Dual-Slope Inductance-to-Digital Converter for Differential Reluctance Sensors, IEEE Trans. Instrum. Meas. 63 (5) (2014) 1364-1371.

[5] S.C. Bera, R. Sarkar, M. Bhowmick, Study of a Modified Differential Inductance Measurement Circuit as Position Transducer of a Power Cylinder, IEEE Trans. Instrum. Meas. 61 (2) (2012) 530-538.

[6] S.C. Bera, N. Mandal, R. Sarkar, Study of a Pressure Transmitter Using an Improved Inductance Bridge Network and Bourdon Tube as Transducer, IEEE Trans. Instrum. Meas. 60 (4) (2011) 1453-1460.

[7] E.G. Bakhoum, M.H.M. Cheng, High-sensitivity inductive pressure sensor, IEEE Trans. Instrum. Meas. 60 (8) (2011) 2960-2966.

[8] Y.H. Kim, S. Hashi, K. Ishiyama, K.I. Arai, M. Inoue, Remote temperature sensing system using reverberated magnetic flux, IEEE Trans. Magnetics 36 (5) (2000) 36433645.

[9] P. Kumar, B. George, V.J. Kumar, A simple signal conditioning scheme for inductive sensors, in: Proceedings of Seventh International Conference on Sensing Technology, 
Wellington, 3-5 Dec. 2013, 512-515.

[10] F. Reverter, J. Jordana, M. Gasulla, R. Pallàs-Areny, Accuracy and resolution of direct resistive sensor-to-microcontroller interfaces, Sensors and Actuators A Phys. 121 (2005) $78-87$.

[11] F. Reverter, O. Casas, Interfacing differential resistive sensors to microcontrollers: a direct approach, IEEE Trans. Instrum. Meas. 58 (10) (2009) 3405-3410.

[12] J. Courbat, D. Briand, L. Yue, S. Raible, N.F. Rooij, Drop-coated metal-oxide gas sensor on polyimide foil with reduced power consumption for wireless applications, Sensors and Actuators B Chem. 161 (2012) 862-868.

[13] F. Reverter, O. Casas, Direct interface circuit for capacitive humidity sensors, Sensors and Actuators A Phys. 143 (2008) 315-322.

[14] F. Reverter, O. Casas, Interfacing differential capacitive sensors to microcontrollers: a direct approach, IEEE Trans. Instrum. Meas. 59 (10) (2010) 2763-2769.

[15] J. Pelegrí-Sebastiá, E. García-Breijo, J. Ibáñez, T. Sogorb, N. Laguarda-Miro, J. Garrigues, Low-cost capacitive humidity sensor for application within flexible RFID labels based on microcontroller systems, IEEE Trans. Instrum. Meas 61 (2) (2012) 545553.

[16] A. Custodio, Contribución al diseño de interfaces de señal en sensores inteligentes, PhD Thesis, Universitat Politècnica de Catalunya, Spain (2001) [In Spanish].

[17] P. Yedamale, J. Bartling, See What You Can Do with the CTMU, AN1375, Microchip Technology Inc. (2011).

[18] Z. Kokolanski, J. Jordana, M. Gasulla, V. Dimcev, F. Reverter, Microcontroller-based interface circuit for inductive sensors, in: Proceedings Eurosensors XXVIII, Brescia, Italy, 7-10 September 2014.

[19] F. Reverter, Z. Kokolanski, J. Jordana, M. Gasulla, Circuito y método para medir sensores inductivos mediante un microcontrolador, Spanish Patent P201430718 (19 May 
2014) [In Spanish].

[20] P. Kejik, C. Kluser, R. Bischofberger, R. S. Popovic, A low-cost inductive proximity sensor for industrial applications, Sensors and Actuators A Phys. 110 (2004) 93-97.

[21] M. R. Nabavi, S. Nihtianov, Design strategies for eddy-current displacement sensor systems: review and recommendations, IEEE Sensors Journal 12 (2012) 3346-3355.

[22] F. Reverter, Power consumption in direct interface circuits, IEEE Trans. Instrum. Meas. 62 (2) (2013) 503-509.

[23] M.I. Rosales, H. Montiel, R. Valenzuela, Magnetic permeability and relaxation frequency in high frequency magnetic materials, Mat. Res. Soc. Symp. Proc. 674 (2001) U1.8.

[24] TDK, Ni-Zn Material Characteristics. Ferrite for coil, (2014) [Online]. Available: http://product.tdk.com/en/catalog/datasheets/ferrite_ni-zn_material_characteristics_en.pdf 


\section{SHORT BIOGRAPHIES}

Zivko KOKOLANSKI was born in Skopje, Macedonia, in 1981. He received B.Sc degree in electronics and telecommunications, M.Sc. degree in electrical measurements and $\mathrm{PhD}$. Degree in electrical measurements from the Ss. Cyril and Methodius University in Skopje, Macedonia, in 2006, 2010 and 2014 respectively. Since 2007 he is with the Department of Electrical Measurements, Faculty of Electrical Engineering and Information Technologies in Skopje, Macedonia, where he is currently an Assistant Professor. In 2010 and 2014 he was granted a fellow award by the International Atomic Energy Agency (IAEA). His research interests include electronic instrumentation, sensor interface circuits, multi-sensor systems and virtual instrumentation.

Josep JORDANA was born in Santa Eulàlia de Ronçana, Spain, on January 8, 1966. He received the Enginyer (MEng) and Doctor Enginyer ( $\mathrm{PhD}$ ) degrees in Telecommunications from the Universitat Politècnica de Catalunya (UPC), Barcelona, in 1990 and 1999, respectively. In 1993, he joined the Department of Electronic Engineering as a Lecturer and currently he is an Associate Professor at the UPC. He is engaged in teaching Analogue and Digital Electronics. His main research interests are in electronic instrumentation, direct sensor-to-microcontroller interfaces, energy harvesting for autonomous sensors and educational research. ORCID ID: 0000-0002-8498-8700.

Manel GASULLA was born in Vinaròs, Spain, on May 26, 1967. He received the Enginyer (MEng) and Doctor Enginyer (PhD) degrees in Telecommunications from the Universitat Politècnica de Catalunya (UPC), Barcelona, in 1992 and 1999, respectively. Since 1993 he has been with the UPC, where he is an associate professor, engaged in teaching on Analog and Power Electronics and Electronic Instrumentation. In 2001-2002 he was a Visiting Postdoctoral Fellow at the Electronic Instrumentation Laboratory, Delft University of Technology, The Netherlands. His research interests include capacitive sensors, direct sensorto-microcontroller interfaces, and energy harvesting and wireless power transfer circuits for autonomous sensors. He is co-author of more than 50 papers in journals and conferences, six Spanish patents, several chapters in books and the book Powering Autonomous Sensors (Springer, 2011). ORCID ID: 0000-0002-0364-6806.

Vladimir DIMCEV obtained B.Sc. in electrical engineering in 1983, M.Sc. in 1990 and Ph.D. in 2001 from Ss. Cyril and Methodius University, Skopje, Macedonia. Since March 1987, he is chair of the Department of Electrical Measurements, Faculty of Electrical Engineering and Information Technologies, University Ss. Cyril and Methodius, Skopje, where he is a Professor. His research areas of interest are: power systems measurements and instrumentation, earthing and measurements of grounding grid parameters, electromagnetic interference, measurements of electromagnetic fields under transmission lines and power transformers, data acquisition systems and power quality. He received award from IEEE Power Engineering Society as distinguished engineer in Region 8 for 2001 and Region 8 section volunteer award for 2011, also a member of the CIGRE - Paris.

Ferran REVERTER was born in Llagostera, Spain, on January 4, 1976. He received the B.Sc. degree in Industrial Electronic Engineering from the University of Girona (Girona, Spain) in 1998, the M.Sc. degree in Electronic Engineering from the University of Barcelona (Barcelona, Spain) in 2001, and the Ph.D. degree in Electronic Engineering from the Universitat Politècnica de Catalunya (UPC) (Barcelona, Spain) in 2004. Since 2001 he has been with the UPC, where he is an Associate Professor in Analogue Electronics and Digital Systems. He was a visiting postdoctoral researcher with the Delft University of Technology (Delft, The Netherlands) from 2005 to 2007, and with the Imperial College London (London, UK) in 2012. His main topics of research are: interface electronics based on microcontrollers for smart sensors, power processing circuits based on DC/DC converters for autonomous sensors, and temperature sensors based on MOSFETs for on-chip thermal testing of ICs. ORCID ID: 0000-0003-1653-0519. 


\section{List of Figure Captions}

Figure 1. (a) Proposed direct interface circuit for an inductive sensor $\left(L_{x}\right)$. (b) Transient response of the voltage at pins 1 and 2 when measuring the RL circuit that includes $L_{x}$. (c) First phase for the measurement of $L_{x}$. (d) Second phase for the measurement of $L_{x}$.

Figure 2. Equivalent circuit to analyse the effect of parasitic resistances on the proposed direct interface circuit.

Figure 3. Quantisation effects on the estimation of $L_{x}$.

Figure 4. Experimental transient response of the voltage at pin 2 during the measurement of the discharging time.

Figure 5. Experimental results when measuring inductances from $1 \mathrm{mH}$ to $10 \mathrm{mH}$ using (a) PIC with $f_{\mathrm{s}}=2.5 \mathrm{MHz}$, (b) PIC with $f_{\mathrm{s}}=5 \mathrm{MHz}$, and (c) AVR with $f_{\mathrm{s}}=16 \mathrm{MHz}$.

Figure 6. Experimental results when measuring inductances from $10 \mathrm{mH}$ to $100 \mathrm{mH}$ using (a) PIC with $f_{\mathrm{s}}=2.5 \mathrm{MHz}$, (b) PIC with $f_{\mathrm{s}}=5 \mathrm{MHz}$, and (c) AVR with $f_{\mathrm{s}}=16 \mathrm{MHz}$.

\section{List of Table Captions}

Table I. Main features of the microcontrollers used to implement the direct interface circuit shown in Fig. 1a. ${ }^{(1)}$ This value has been calculated by (8) assuming $R_{0}=99.0 \Omega$.

Table II. Theoretical and experimental value of the coefficients of the quadratic response that relates $L_{x}^{*}$ versus $L_{x}$ for the three cases under test. Theoretical values are obtained from (12) using the data in Table I, whereas the experimental ones result from fitting a quadratic polynomial to the experimental data in Fig. 6. 
Figure 1

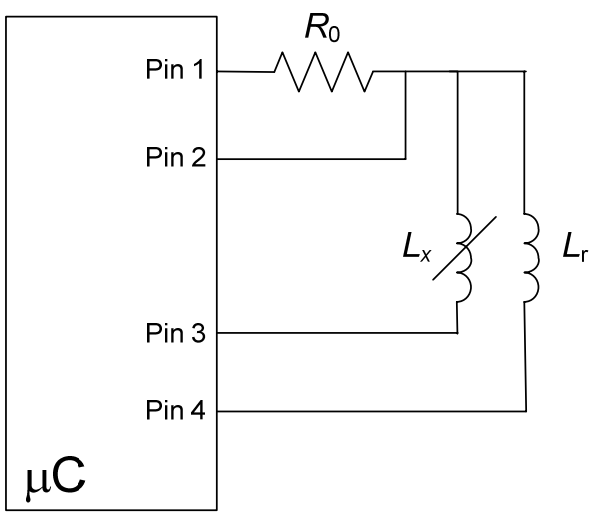

(a)

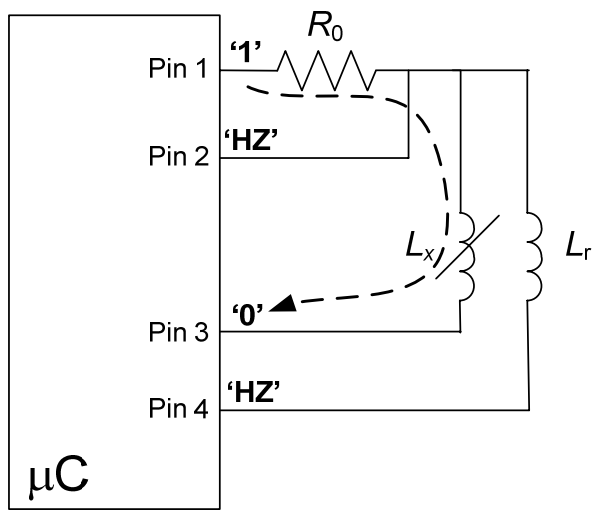

(c)

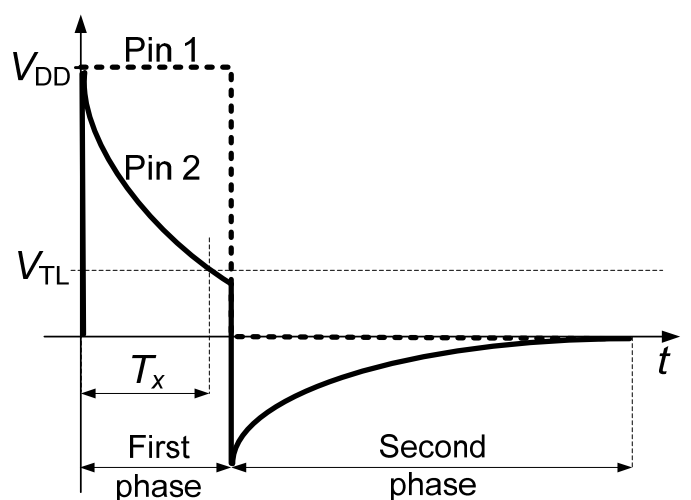

(b)

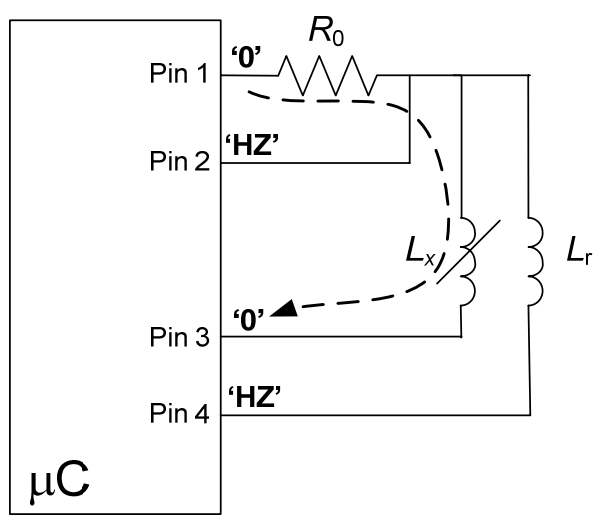

(d) 
Figure 2

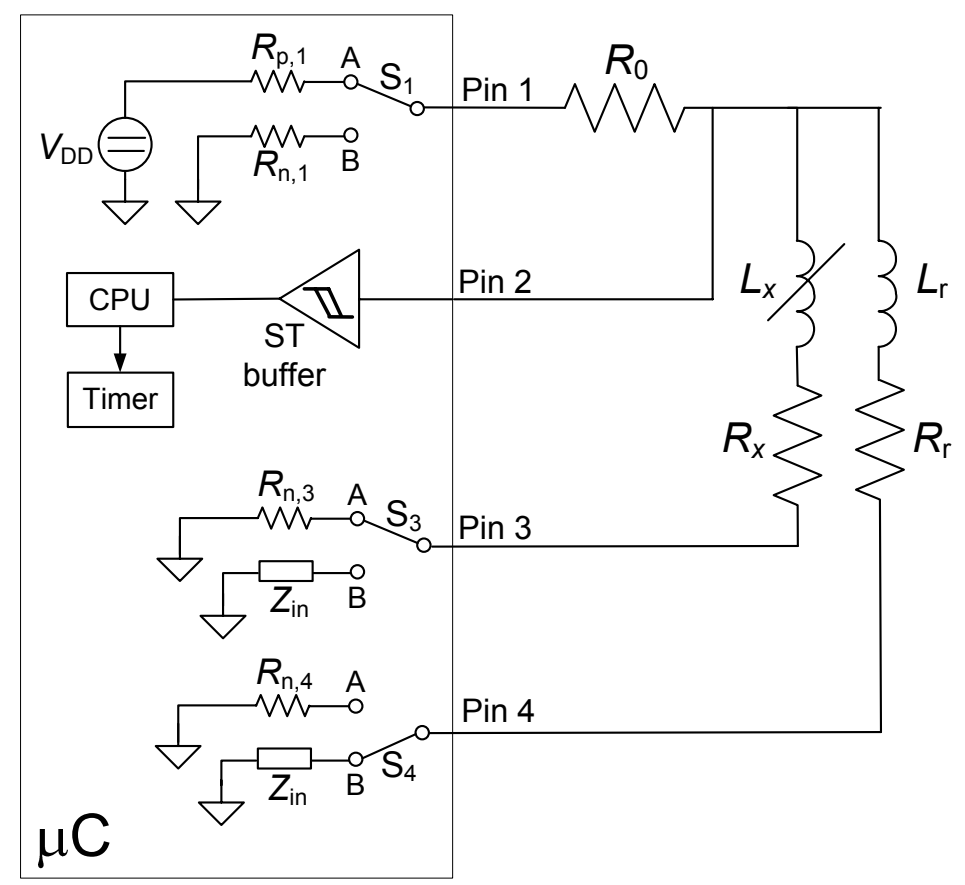


Figure 3

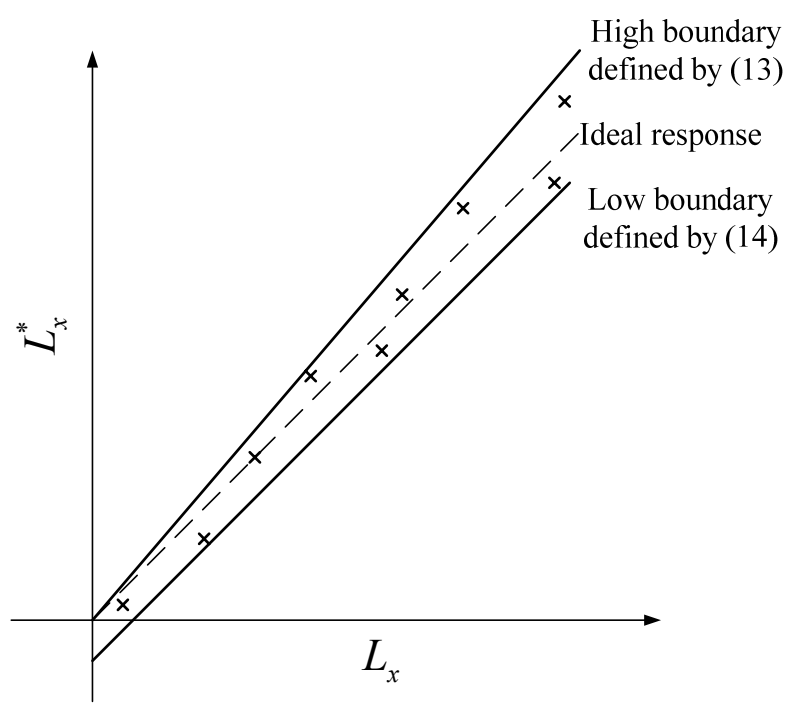

Figure 4

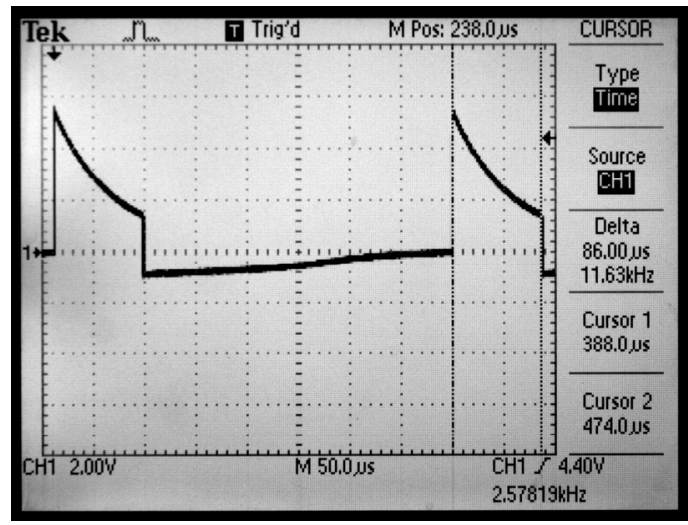


Figure 5

(a)

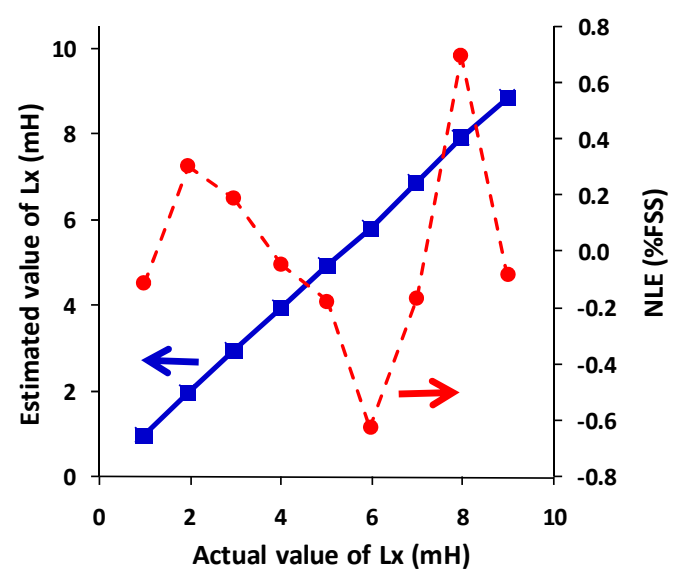

(b)

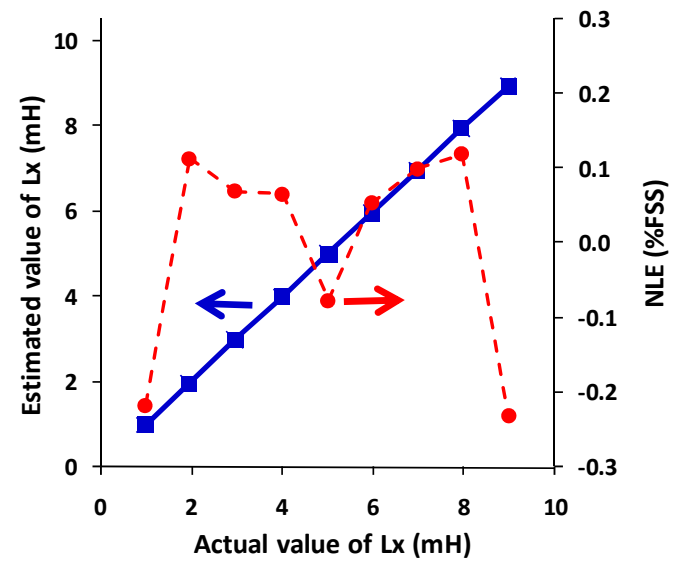

(c)

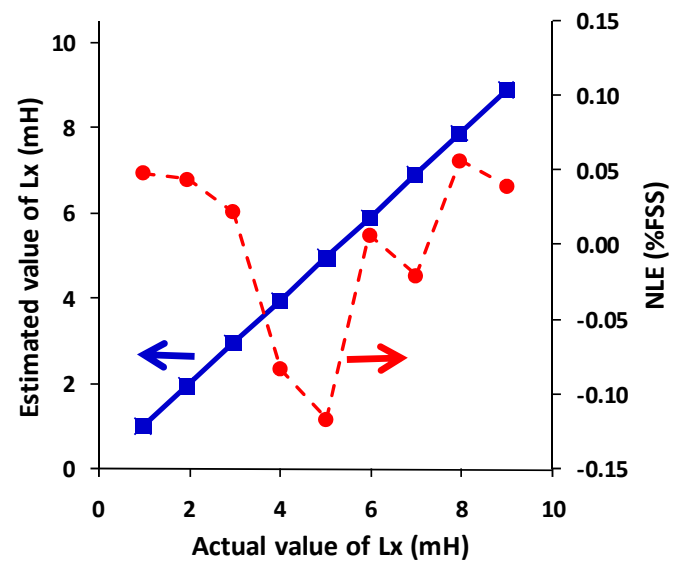


Figure 6

(a)

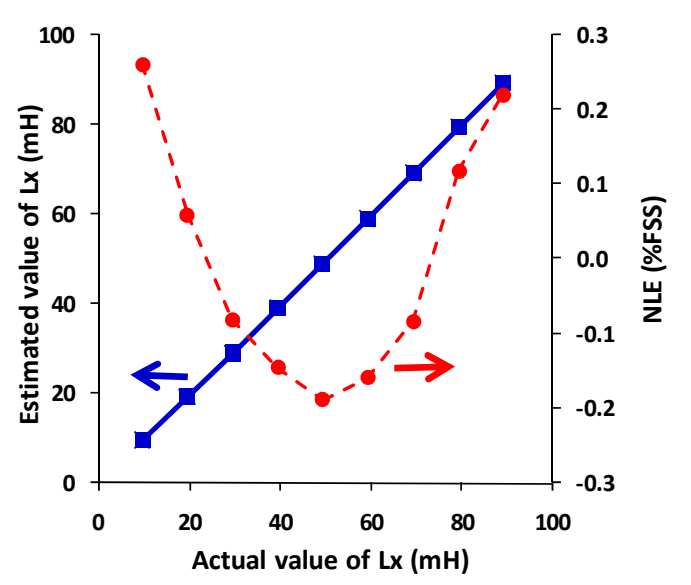

(b)

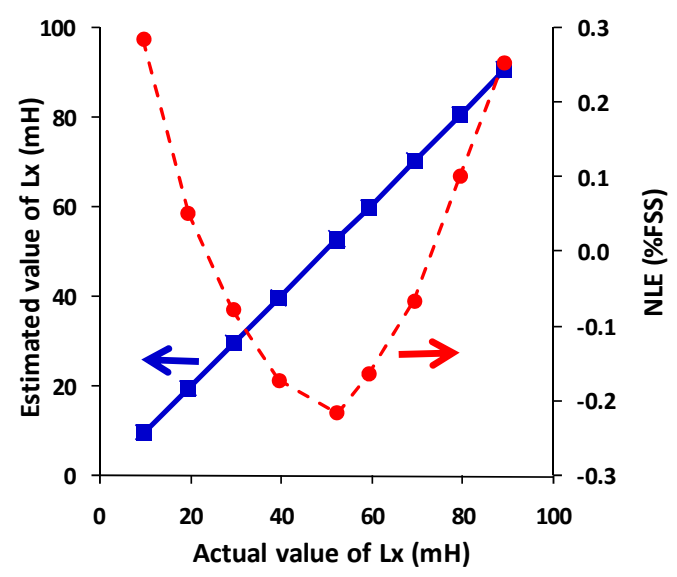

(c)

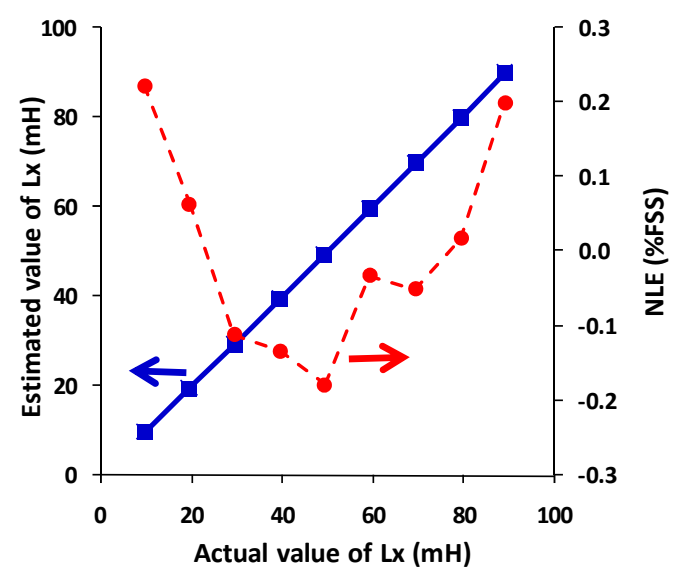


Table I

\begin{tabular}{|c|c|c|}
\hline Feature & $\begin{array}{c}\text { PIC16F877 } \\
\text { (Microchip) }\end{array}$ & $\begin{array}{c}\text { AVR ATmega328P } \\
\text { (Atmel) }\end{array}$ \\
\hline$f_{\mathrm{s}}(\mathrm{MHz})$ & 2.5 and 5 & 16 \\
\hline$V_{\mathrm{DD}}(\mathrm{V})$ & 5.06 & 5.06 \\
\hline$V_{\mathrm{TL}}(\mathrm{V})$ & 1.50 & 2.42 \\
\hline$R_{\mathrm{p}, 1}(\Omega)$ & 83.9 & 26.2 \\
\hline$R_{\mathrm{n}, 3}(\Omega)$ & 29.8 & 23.1 \\
\hline$R_{\mathrm{n}, 4}(\Omega)$ & 29.1 & 23.2 \\
\hline$\alpha^{(1)}\left(\Omega^{-1}\right)$ & 0.0052 & 0.0038 \\
\hline
\end{tabular}

Table II

\begin{tabular}{|c|c|c|c|c|c|c|}
\cline { 2 - 7 } \multicolumn{1}{c|}{} & \multicolumn{2}{c|}{ PIC at $2.5 \mathrm{MHz}$} & \multicolumn{2}{c|}{ PIC at $5 \mathrm{MHz}$} & \multicolumn{2}{c|}{ AVR at $16 \mathrm{MHz}$} \\
\cline { 2 - 7 } \multicolumn{1}{c|}{} & Theoretical & Experim. & Theoretical & Experim. & Theoretical & Experim. \\
\hline $\begin{array}{c}\text { Constant } \\
\text { term }\end{array}$ & 0 & -0.10 & 0 & -0.01 & 0 & -0.04 \\
\hline $\begin{array}{c}\text { Linear } \\
\text { coefficient }\end{array}$ & 0.99 & 0.98 & 0.99 & 0.99 & 0.99 & 0.99 \\
\hline $\begin{array}{c}\text { Quadratic } \\
\text { coefficient }\end{array}$ & $2.3 \cdot 10^{-4}$ & $2.8 \cdot 10^{-4}$ & $2.3 \cdot 10^{-4}$ & $3.0 \cdot 10^{-4}$ & $1.7 \cdot 10^{-4}$ & $2.2 \cdot 10^{-4}$ \\
\hline
\end{tabular}

